\title{
Motor gerador ciclo diesel assistido por sistemas de automação industrial (Indústria
}

\section{0)}

\author{
Diesel cycle generator engine assisted by industrial automation systems (Industry 4.0) \\ Motor generador de ciclo diésel asistido por sistemas de automatización industrial (Industria 4.0)
}

Recebido: 13/12/2021 | Revisado: 21/12/2021 | Aceito: 24/12/2021 | Publicado: 04/01/2022

Vander Fabio Silveira

ORCID: https://orcid.org/0000-0002-5725-0591

Universidade Estadual do Oeste do Paraná, Brasil

E-mail: vfabios@hotmail.com

Jair Antonio Cruz Siqueira

ORCID: https://orcid.org/0000-0002-8140-444X

Universidade Estadual do Oeste do Paraná, Brasil

E-mail: jair.siqueira@unioeste.br

Reginaldo Ferreira Santos

ORCID: https://orcid.org/0000-0002-7745-9173

Universidade Estadual do Oeste do Paraná, Brasil

E-mail: reginado.santos@unioeste.br

Fernando de Lima Caneppele

ORCID: https://orcid.org/0000-0003-4498-8682

Universidade de São Paulo, Brasil E-mail: caneppele@usp.br

Jonathan Dieter

ORCID: https://orcid.org/0000-0002-3674-4955 Universidade Federal do Paraná, Brasi E-mail: jony.dieter@gmail.com

Maritane Prior

ORCID: https://orcid.org/0000-0002-3232-1533

Universidade Estadual do Oeste do Paraná, Brasil

E-mail: maritane.prior@unioeste.br

Luciene Kazue Tokura

ORCID: https://orcid.org/0000-0001-9758-0141

Universidade Estadual do Oeste do Paraná, Brasil

E-mail: lucienetokura@gmail.com

Gilson Debastiani

ORCID: https://orcid.org/0000-0003-3429-4257

Universidade Estadual do Oeste do Paraná, Brasil

E-mail: gilsondebastiani2013@gmail.com

Cristiano Fernando Lewandoski

ORCID: https://orcid.org/0000-0001-5944-5723

Universidade Estadual do Oeste do Paraná, Brasil

E-mail: cristiano@aceletric.com

Leonardo da Silva Reis

ORCID: https://orcid.org/0000-0002-9239-6158

Universidade Estadual do Oeste do Paraná, Brasil

E-mail: leonardoreis@hotmail.com

\begin{abstract}
Resumo
Combustíveis como o biodiesel vêm ganhando muita popularidade pelo fato de ser um combustível alternativo. O estudo trata da avaliação do desempenho e de emissões de um motor gerador operando com diesel e blendas de biodiesel, assistido por sistema de automação industrial utilizados na Indústria 4.0. O experimento foi conduzido nos laboratórios da Universidade Estadual do Oeste do Paraná. Foram avaliados durante o experimento a energia gerada, o consumo específico, a eficiência energética e as emissões geradas pelo conjunto motor gerador. Os tratamentos utilizados foram o diesel de petróleo tipo A (D100), cinco blendas (misturas) de biodiesel de canola (B5, B10, B15, B20 e B50) e biodiesel puro (B100). As cargas aplicadas ao motor gerador foram do tipo resistivas de 1,0; 1,5; 4,5 e 6,0 kW para cada tipo de combustível. A energia gerada se manteve crescente e estável conforme o aumento de carga. O melhor resultado do consumo específico foi com o diesel (D100), seguido por B10 e o B20, ambos para a carga de 4,5 kW. As emissões de gases de monóxido de carbono são reduzidas enquanto as emissões de dióxido de carbono aumentam com aplicação de maiores cargas. O biodiesel puro (B100) tende a apresentar melhor eficiência energética que as misturas binárias
\end{abstract}


utilizadas quando inserida a carga de $6,0 \mathrm{~kW}$. Os resultados obtidos demonstram que as misturas de biodiesel de canola com o diesel convencional são uma das possíveis soluções viáveis na substituição parcial do diesel mineral.

Palavras-chave: Biodiesel; Geração de energia; Gases de efeito estufa.

\begin{abstract}
Fuels such as biodiesel have been gaining popularity as an alternative fuel. The study deals with the evaluation of the performance and emissions of a generator engine operating with diesel and biodiesel blends, assisted by an industrial automation system used in Industry 4.0. The experiment was carried out in the laboratories of the State University of West Paraná. The energy generated, the specific consumption, the energy efficiency and the emissions generated by the generator set were evaluated during the experiment. The treatments used were type A petroleum diesel (D100), five blends (mixtures) of rapeseed biodiesel (B5, B10, B15, B20 and B50) and pure biodiesel (B100). The loads applied to the generator motor were of the 1.0 resistive type; $1.5 ; 4.5$ and $6.0 \mathrm{~kW}$ for each fuel type. The generated energy kept increasing and stable as the load increased. The best result of specific consumption was with diesel (D100), followed by B10 and B20, both for the $4.5 \mathrm{~kW}$ load. Carbon monoxide gas emissions are reduced while carbon dioxide emissions increase with application of higher loads. Pure biodiesel (B100) tends to have better energy efficiency than the binary mixtures used when inserted at a load of $6.0 \mathrm{~kW}$. The results obtained demonstrate that the blends of rapeseed biodiesel with conventional diesel are one of the possible viable solutions for the partial replacement of mineral diesel.
\end{abstract}

Keywords: Biodiesel; Power generation; Greenhouse gases.

\title{
Resumen
}

Los combustibles como el biodiésel han ido ganando popularidad como combustible alternativo. El estudio trata de la evaluación del rendimiento y las emisiones de un motor generador que funciona con mezclas de diésel y biodiésel, asistido por un sistema de automatización industrial utilizado en la Industria 4.0. El experimento se llevó a cabo en los laboratorios de la Universidad Estatal de West Paraná. Durante el experimento se evaluó la energía generada, el consumo específico, la eficiencia energética y las emisiones generadas por el grupo electrógeno. Los tratamientos utilizados fueron diesel de petróleo tipo A (D100), cinco mezclas (mezclas) de biodiésel de colza (B5, B10, B15, B20 y B50) y biodiésel puro (B100). Las cargas aplicadas al motor del generador fueron del tipo resistivo 1.0; 1,$5 ; 4,5$ y 6,0 kW para cada tipo de combustible. La energía generada siguió aumentando y estable a medida que aumentaba la carga. El mejor resultado de consumo específico fue con diésel (D100), seguido de B10 y B20, ambos para la carga de 4,5 $\mathrm{kW}$. Las emisiones de gas de monóxido de carbono se reducen mientras que las emisiones de dióxido de carbono aumentan con la aplicación de cargas más altas. El biodiésel puro (B100) tiende a tener una mejor eficiencia energética que las mezclas binarias utilizadas cuando se inserta a una carga de $6,0 \mathrm{~kW}$. Los resultados obtenidos demuestran que las mezclas de biodiesel de colza con diesel convencional son una de las posibles soluciones viables para la sustitución parcial del diesel mineral.

Palabras clave: Biodiesel; Generación de energía; Gases de invernadero.

\section{Introdução}

O biodiesel é um dos combustíveis alternativos promissores para motores diesel, que ganhou popularidade nas últimas décadas. É constituído principalmente por ésteres metílicos e etílicos de ácidos graxos (triglicerídeos), principalmente derivados de óleos vegetais comestíveis e não comestíveis e gorduras animais através do processo de transesterificação, onde os triglicerídeos reagem com o álcool de cadeia curta na presença de um catalisador. (Yesilyurt \& Cesur, 2020)

O biodiesel pode ser produzido a partir de uma variedade de matérias-primas, tais como, canola, soja, girassol, mamona e etc. (Aldhaidhawi, Chiriac, Badescu \& Delay, 2017; Saifuddin, Refal \& Kumaran, 2017).

A canola (Brassica napus L. var. oleífera) é uma espécie oleaginosa da família Brassicaceae, sendo a segunda maior cultura oleaginosa produzida em todo o mundo. A colza inicialmente com alto teor de ácido erúcico, foi desenvolvida por pesquisadores canadenses, onde identificaram uma semente de colza com baixo teor de ácido erúcico $(<2 \%)$ e a chamaram de canola (para significar óleo do Canadá ou óleo do Canadá com baixo teor de ácido) (Assefa et al., 2018). Desta forma, este estudo propõe o óleo de canola como matéria-prima que através do processo de transesterificação obtém-se o biocombustível denominado biodiesel.

O biodiesel é um combustível renovável, biodegradável, não tóxico e com propriedades comparáveis às do diesel de origem mineral. Além de ser mais vantajoso quando comparado ao diesel devido ao seu ponto de inflamação mais elevado, menor volatilidade e maior lubricidade (Noor et al., 2016). Apresenta ainda um teor de compostos aromáticos mais baixos, 
Research, Society and Development, v. 11, n. 1, e20611124699, 2022

(CC BY 4.0) | ISSN 2525-3409 | DOI: http://dx.doi.org/10.33448/rsd-v11i1.24699

contém de 10 a $12 \%$ de oxigênio em peso e ausência de enxofre. Devido à maior presença de oxigênio, é alcançado um maior grau de oxidação de hidrocarbonetos queimados na câmara do motor, que são gerados durante a combustão (Agarwal, Gupta, Dixit \& Shukla, 2013). Isso leva a menores emissões de monóxido de carbono (CO), hidrocarbonetos não queimados (HC) e material particulado (PM) para combustível a base de biodiesel. Entretanto, um maior teor de oxigênio no biodiesel produz emissões mais elevadas de óxido nitroso $\left(\mathrm{NO}_{\mathrm{x}}\right)$, o que constitui uma grande preocupação (Rahman, Rasul, Hassan, Azad, \& Uddin, 2017). Outra desvantagem é a sua maior viscosidade (Nayak, Pattanaik \& Nayak , 2014; Radha, Naga, Rajagopal \& Nagesh, 2011; No, 2011). A maior viscosidade do biodiesel leva a problemas de bombeamento, atomização deficiente, redução de fluxo do combustível, entupimento de injetores, obstrução de filtros, aumento de depósitos de carbono na cabeça do pistão, etc (No, 2014; Sundararajan, Subbiah \& Gomathinayakam, 2016). Podem apresentar ainda menor volatilidade, poder calorifico, densidade de energia e maior consumo especifico de combustível (Singh, Kumar \& Muk, 2012).

Para minimizar os pontos indesejáveis, misturas de diesel mineral e biodiesel apresentam uma alternativa viável. Hasan et al. (2015) relataram que o combustível misturado com biodiesel apresenta menor torque e maior consumo específico do que o combustível diesel devido aos menores valores do poder caloríficos contidos no biodiesel.

O biodiesel proveniente da canola é uma alternativa para uso em motogeradores em blendas ou misturas entre biodiesel e diesel mineral. Sendo analisados neste estudo sob aplicação de cargas resistivas, verificou-se o desempenho de um conjunto motor gerador assistido através de equipamentos utilizados em automação industrial e indústria 4.0.

Diante do exposto, o objetivo do estudo foi avaliar o consumo específico, a eficiência energética e as emissões geradas para o diesel e as diferentes blendas de biodiesel de canola.

\section{Material e Métodos}

A metodologia se baseou em um estudo de caso (Severino, 2017) do desempenho de um gerador ciclo diesel com diferentes blendas de biodiesel de canola.

Neste estudo utilizou-se um motogerador ciclo diesel BD-8500 E3 acionado por um motor de 13,0 cv, acoplado a um gerador com 7,5 kVA/6,0 kW de potência nominal, com tensão de saída de $240 \mathrm{~V}$ trifásico. O biodiesel utilizado foi produzido em pequenos lotes através do processo de transesterificação via rota metílica no laboratório multifuncional CTA - Centro de Tecnologia Automotiva da UNIOESTE, na cidade de Cascavel. O diesel puro (tipo A - S10) foi doado por uma distribuidora local de combustíveis.

Para efetuar o registro dos dados durante todo o experimento foram utilizados sistemas de aquisição e registro de dados com padrão industrial (Indústria 4.0). Para isto, empregou-se um controlador lógico programável (CLP) marca Eaton, modelo XV-102-D6-70TWR, dotado de uma interface homem-máquina (IHM) com touch screen de 7" (sete polegadas), uma unidade terminal remota (RTU) modelo XN-GWBR-CANopen marga Eaton, um medidor multifuncional de parâmetros elétricos modelo DPM-C520 marca Delta e um indicador de pesagem com célula de carga modelo 3107C marca Alfa. Para conectar os equipamentos foram utilizados protocolos de redes industriais (Modbus-RS485 e CAN-open) apresentando as informações em tempo real do processo em um sistema supervisório, a fim de monitorar, apresentar, registrar e armazenar os preceitos do sistema de geração. A carga utilizada consistiu em um banco de quinze resistências aletadas com dissipação nominal de $1500 \mathrm{~W} / 220 \mathrm{~V}$ cada perfazendo um total de $6 \mathrm{~kW}$. Um resumo geral da montagem pode ser observado na Figura 1. 
Figura 1. Equipamentos com padrão industrial utilizado para monitorar e controlar o sistema motor gerador.

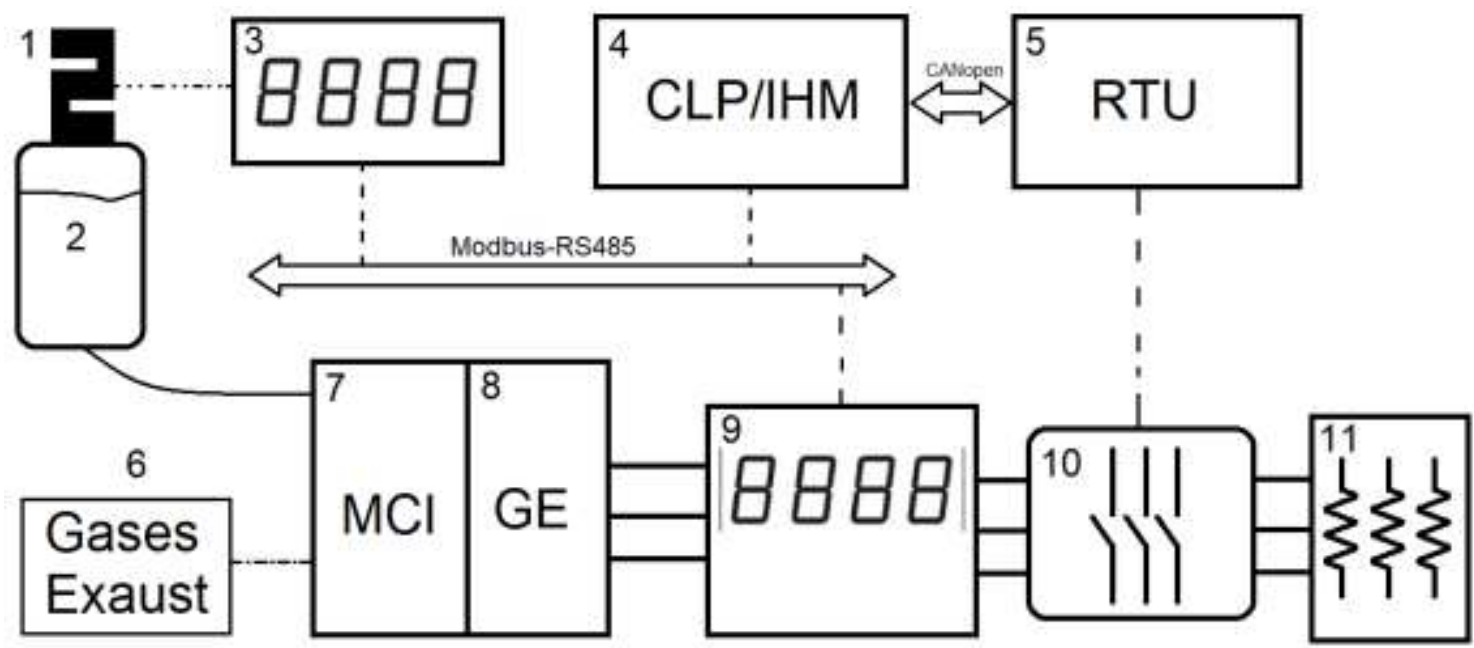

1: Célula de carga, 2: Tanque de combustível, 3: Indicador de pesagem, 4: Controlador lógico programável/Interface homem máquina, 5: Unidade terminal remota, 6: Analisador de gases (PCA-3), 7: Motor diesel, 8: Gerador elétrico, 9: Multimedidor de parâmetros elétricos, 10: Contatores, 11: Cargas resistivas. Fonte: Autores.

O combustível utilizado foi composto por blendas ou misturas volumétricas entre biodiesel de canola e óleo diesel tipo A-S10 (baixo teor de enxofre - $10 \mathrm{mg} / \mathrm{kg}$ ). A proporção do biodiesel na composição do combustível foi de 0\% (D100 - diesel mineral), 5\% (B5), 10\% (B10), 15\% (B15), 20\% (B20), 50\% (B50) e 100\% de biodiesel (B100). Para medir a massa de combustível consumida foi utilizado um tanque externo de armazenamento, fixado em uma célula de carga, para obter o consumo de combustível nos testes do conjunto motogerador.

Na Equação 1 pode ser observada o cálculo do consumo de combustível, para cada teste de desempenho do conjunto motogerador.

$$
\mathrm{Ma}=(\mathrm{mi}-\mathrm{mf}) / \mathrm{t}
$$

Onde: Ma - consumo horário de combustível (g/s); mi - massa inicial de combustível (g); mf - massa final de combustível (g); $\mathrm{t}$ - tempo de ensaio (s).

O consumo específico de combustível foi determinado utilizando a unidade $\left(\mathrm{g} \cdot \mathrm{kW}^{-1} \mathrm{~h}^{-1}\right)$, conforme a Equação 2.

$$
\mathrm{CE}=(3600 * \mathrm{Ma}) / \mathrm{EG}
$$

Onde: $\mathrm{CE}$ - consumo específico $\left(\mathrm{g} \cdot \mathrm{kW}^{-1} \mathrm{~h}^{-1}\right) ; \mathrm{Ma}$ - consumo horário volumétrico $\left(\mathrm{kg} . \mathrm{s}^{-1}\right)$; e EG - energia gerada no conjunto motor gerador $(\mathrm{kW})$.

A eficiência energética foi calculada por meio da Equação 3.

$$
\mathrm{EE}=[3600 /(\mathrm{PCI} * \mathrm{CE})]^{*} 100
$$

$\mathrm{Na}$ qual: EE - eficiência energética (\%); PCI - poder calorífico inferior do combustível (MJ.kg-1); CE - consumo específico do combustível $\left(\mathrm{g} \cdot \mathrm{kW}^{-1} \mathrm{~h}^{-1}\right)$; e 3600 - fator de conversão entre unidades. 
Para a determinação e análise dos gases de combustão foi utilizado um analisador de gás marca Bacarach modelo PCA3. O poder calorífico inferior (PCI) dos combustíveis foi determinado segundo a Equação 4.

$$
\mathrm{PCI}=\mathrm{PCS}-3,052
$$

Onde: PCI - poder calorífico inferior (MJ.Kg $\left.{ }^{-1}\right)$; PCS - poder calorífico superior $\left(\mathrm{MJ}_{\mathrm{Kg}}{ }^{-1}\right)$.

A análise dos gases de combustão gerados no ensaio do conjunto motogerador usando combustíveis com misturas à base de óleo vegetal foi realizada com o uso do analisador portátil de gases de combustão da marca Bacharach, modelo PCA3. $\mathrm{O}$ aparelho possui sensores eletroquímicos capazes de realizar as medições de oxigênio $\left(\mathrm{O}_{2}\right)$, monóxido de carbono $(\mathrm{CO})$, óxido de nitrogênio (NO), Dióxido de nitrogênio $\left(\mathrm{NO}_{2}\right)$ e calcular os valores de óxidos de nitrogênio (NOx).

\section{Resultados e Discussão}

\subsection{Consumo específico}

O comportamento do consumo específico (CE) do sistema com motor gerador em função das cargas aplicadas pode ser observado na Figura 2, apresentando CE maior para as cargas menores (1,0 e 1,5 kW). Com o uso do óleo diesel AS-10 (D100), as médias do CE do motogerador foram ligeiramente menores, em relação as blendas com biodiesel B5, B10, B15, B20, B50 e B100 (Figura 2).

Segundo pesquisas realizadas por Gharehghani, Mirsalim e Hosseini (2017) também observaram comportamentos semelhantes quando adicionado biodiesel com diesel, apresentando consumo especifico superior em maiores proporções de mistura. As blendas biodiesel-diesel e o diesel AS-10 apresentaram comportamento semelhante, com redução do CE para as cargas 1,0; 1,5 e 4,5 kW e um pequeno aumento na de $6 \mathrm{~kW}$ (Figura 2). Este comportamento de redução no CE em cargas intermediarias e aumento nas cargas próxima da nominal do sistema também foi observado nos estudos realizados por (Simsek, 2020).

Entre as diferentes blendas utilizadas no estudo, a que resultou no menor CE foi B10 (Figura 2B) quando aplicada a carga nominal de 4,5 kW, apresentando um CE de 345,30 g. $\mathrm{kW}^{-1} \mathrm{~h}^{-1}$. Enquanto o CE do diesel D100 para a mesma carga apresentou 342,27 g.kW ${ }^{-1} \mathrm{~h}^{-1}$. Já quando aplicado o biocombustível B100, foi registrado um CE de 430,33 g.kW-1 $\mathrm{h}^{-1}$ (Figura 2F) Tal comportamento foi observado por Xue, Grift e Hansen (2011) em estudos com motores ciclo diesel, quando alimentados por biocombustível apresentou maior CE do biodiesel, e o CE foi menor quando houve diminuição da mistura de biodiesel ao diesel convencional. Observa-se ainda que o maior CE ocorreu na blenda B50 ao aplicar a menor carga $(1,0 \mathrm{~kW})$, com valores de 699,32 g.kW-1 $\mathrm{h}^{-1}$ (Figura 2E), enquanto o menor CE ocorreu na carga de 4,5 $\mathrm{kW}$ com 345,30 g.kW $\mathrm{kW}^{-1} \mathrm{~h}^{-1}$ quando abastecido com combustível B10 (Figura 2B).

Serdar, Can e Ozt (2017) descrevem em seus estudos com blendas de biodiesel de canola (5, 10, 15 e $20 \%$ em volume) com óleo diesel, ao alimentar um motor monocilíndrico aplicado em diferentes cargas, observaram aumento do CE ao elevar a mistura de biocombustível, corroborando com os resultados encontrados neste trabalho. 
Figura 2. Avaliação do sistema motor gerador pelo Consumo específico
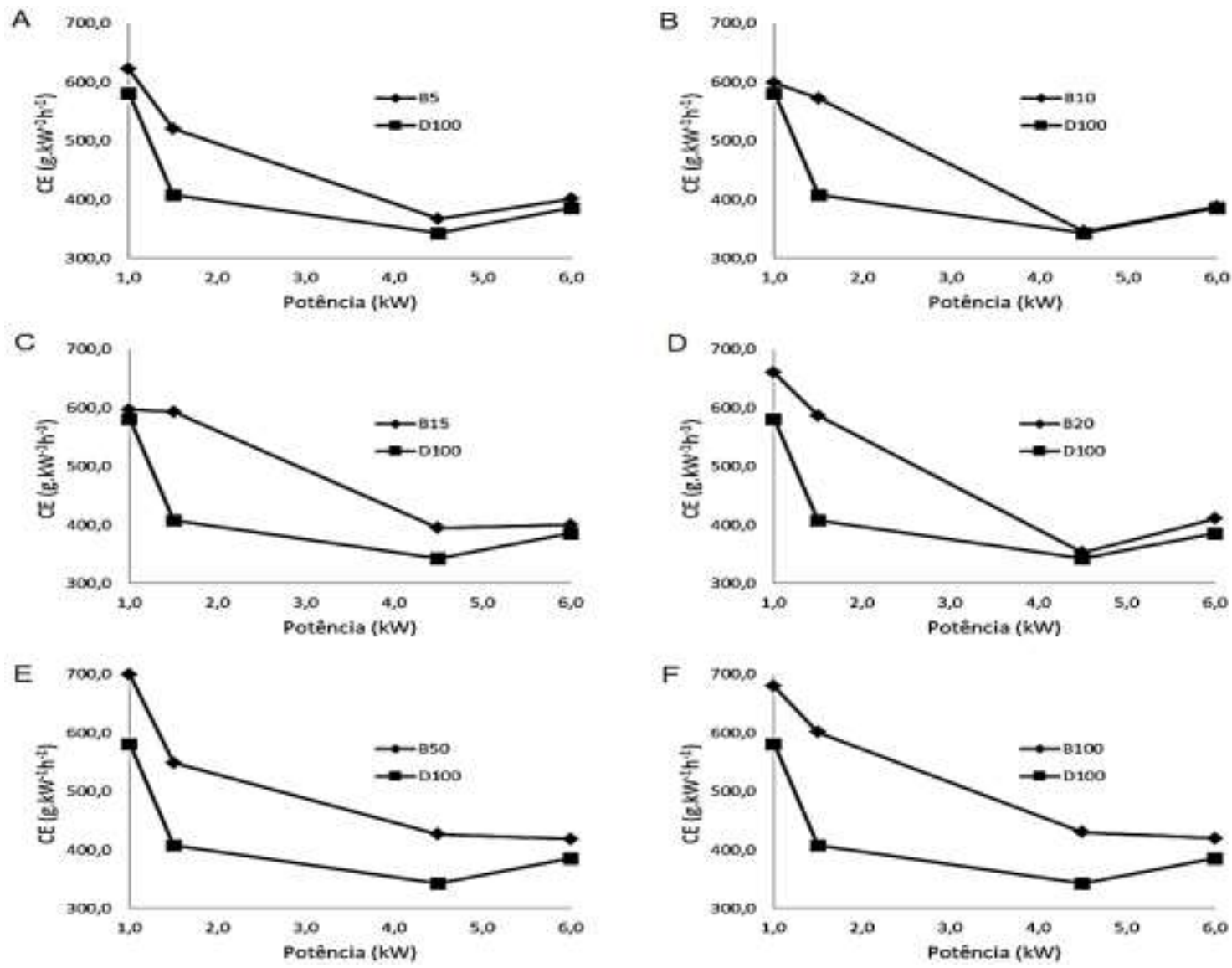

Fonte: Autores.

\subsection{Eficiência energética}

A eficiência na conversão da energia quimicamente contida no combustível em energia elétrica, realizada pelo motogerador foi coletada para as seis blendas de biodiesel e diesel, aplicadas para todas as cargas resistivas e podem ser observadas na Figura 3.

A maior eficiência obtida foi para o sistema foi registrado em 25,52\%, quando alimentado por biodiesel B10 (Figura 3B), e 25,35\% quando operado com mistura B20 (Figura 3D), ambos com carga efetiva de 4,5 kW. Quando o conjunto foi conduzido com as blendas combustíveis B5, B10, B15 e B20 para a carga de 6,0 kW houve tendência de redução na eficiência conforme pode ser observado nas Figuras 3A, 3B, 3C e 3D com valores de 21,84; 22,72; 22,20 e 21,79\%, respectivamente. Quando comparadas a carga de 4,5 kW apresentou 23,86; 25,52; 22,51 e 25,35\%, respectivamente aos mesmos combustíveis, portanto, aumento de eficiência. 
Figura 3. Avaliação do sistema moto gerador pela eficiência energética.
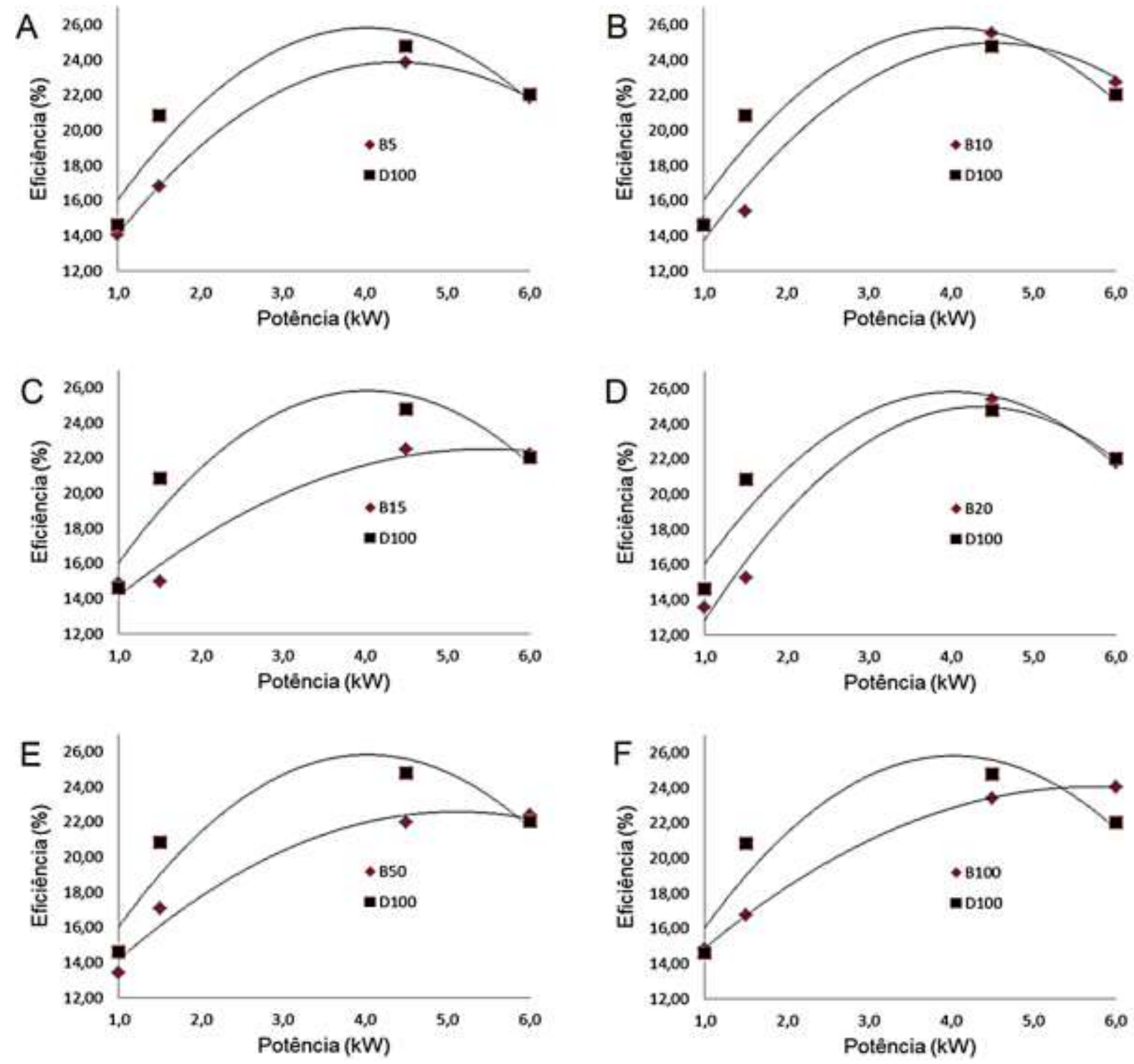

Fonte: Autores.

\subsection{Emissões avaliadas}

Foram coletados dados de emissões para os gases monóxido de carbono $(\mathrm{CO})$, dióxido de carbono $\left(\mathrm{CO}_{2}\right)$ e óxido nitroso (NOx).

As emissões de monóxido de carbono (CO) dependem do conteúdo de oxigênio, do conteúdo de carbono e da eficiência de combustão do combustível aplicado ao motor. Durante a combustão, o carbono presente no combustível sofre reações de oxidação e redução. O teor de carbono contido no combustível oxida com o oxigênio presente no ar produzindo CO e, posteriormente, em $\mathrm{CO}_{2}$. Se houver menor disponibilidade de oxigênio, isso causará a combustão incompleta do combustível utilizado, liberando, consequentemente, maiores taxas de CO (Sakthivel, Nagarajan, Ilangkumaran \& Bajirao, 2014). Para motores a diesel, um dos parâmetros que determina a performance da combustão de determinado combustível será a emissão de $\mathrm{CO}_{2}$, indicando que o hidrocarboneto atingiu sua combustão completa (Erdo, 2020). As variações das emissões de monóxido de carbono $(\mathrm{CO})$ e dióxido de carbono $\left(\mathrm{CO}_{2}\right)$ podem ser observados nas Tabelas 1 e 2, respectivamente. 
$\mathrm{O}$ óxido nitroso (NOx) é composto por monóxido de nitrogênio (NO) associado com dióxido de nitrogênio $\left(\mathrm{NO}_{2}\right)$. Sua formação é comum em motores alimentados por combustível diesel, sendo um fenômeno complexo, e depende de três fatores importantes: temperatura de combustão, concentração de oxigênio e atraso da ignição (Qi, Chen, Geng \& Bian, 2010).

Diversos autores relatam aumento nas emissões de óxido nitroso (NOx) com aumento da carga e misturas de biodiesel ao diesel mineral (Serdar et al., 2017; Dharma, Haji, Chyuan \& Hanra, 2017; Mohammad, Miri, Reza, Seyedi \& Ghobadian, 2017).

Nas Tabelas 1, 2 e 3 apresentam os dados da análise estatística dos gases gerados: $\mathrm{CO}, \mathrm{CO}_{2}$ e NOx, respectivamente, para as blendas dos combustíveis testados, onde as médias seguidas da mesma letra não diferem significativamente pelo teste de Tukey.

Tabela 1. Análise estatística CO.

\begin{tabular}{ccccc}
\hline Tratamento & $\mathbf{1 , 0} \mathbf{k W}$ & $\mathbf{1 , 5} \mathbf{k W}$ & $\mathbf{4 , 5} \mathbf{k W}$ & $\mathbf{6 , 0} \mathbf{k W}$ \\
\hline B5 & $1017,35 \mathrm{a}$ & $829,70 \mathrm{a}$ & $412,32 \mathrm{c}$ & $516,90 \mathrm{c}$ \\
B10 & $774,61 \mathrm{c}$ & $668,00 \mathrm{c}$ & $339,48 \mathrm{~d}$ & $630,93 \mathrm{c}$ \\
B15 & $758,22 \mathrm{c}$ & $671,00 \mathrm{c}$ & $416,12 \mathrm{c}$ & $978,41 \mathrm{a}$ \\
B20 & $685,58 \mathrm{~d}$ & $608,58 \mathrm{~d}$ & $447,29 \mathrm{~b}$ & $977,35 \mathrm{a}$ \\
B50 & $525,41 \mathrm{e}$ & $496,64 \mathrm{e}$ & $343,09 \mathrm{~d}$ & $835,61 \mathrm{~b}$ \\
B100 & $474,12 \mathrm{f}$ & $391,96 \mathrm{f}$ & $235,83 \mathrm{e}$ & $794,48 \mathrm{~b}$ \\
D100 & $864,41 \mathrm{~b}$ & $763,51 \mathrm{~b}$ & $561,93 \mathrm{a}$ & $1000,93 \mathrm{a}$ \\
\hline CV $(\%)$ & 6,3 & 4,11 & 10,56 & 18,82 \\
\hline
\end{tabular}

Médias seguidas da mesma letra não diferem significativamente pelo teste de Tukey. Fonte: Autores.

Tabela 2. Análise estatística $\mathrm{CO}_{2}$.

\begin{tabular}{ccccc}
\hline Tratamento & $\mathbf{1 , 0} \mathbf{k W}$ & $\mathbf{1 , 5} \mathbf{k W}$ & $\mathbf{4 , 5} \mathbf{k W}$ & $\mathbf{6 , 0} \mathbf{k W}$ \\
\hline B5 & $4,00 \mathrm{~b}$ & $3,99 \mathrm{c}$ & $7,05 \mathrm{bc}$ & $9,12 \mathrm{~cd}$ \\
B10 & $3,89 \mathrm{c}$ & $3,89 \mathrm{a}$ & $7,02 \mathrm{c}$ & $9,29 \mathrm{c}$ \\
B15 & $3,84 \mathrm{~d}$ & $4,01 \mathrm{c}$ & $7,16 \mathrm{~b}$ & $9,24 \mathrm{~cd}$ \\
B20 & $4,10 \mathrm{a}$ & $4,06 \mathrm{~b}$ & $7,34 \mathrm{a}$ & $9,49 \mathrm{~b}$ \\
B50 & $3,80 \mathrm{e}$ & $4,09 \mathrm{ab}$ & $7,36 \mathrm{a}$ & $9,84 \mathrm{a}$ \\
B100 & $3,80 \mathrm{e}$ & $4,10 \mathrm{a}$ & $7,43 \mathrm{a}$ & $9,86 \mathrm{a}$ \\
D100 & $3,86 \mathrm{~d}$ & $3,86 \mathrm{a}$ & $6,99 \mathrm{c}$ & $9,05 \mathrm{~d}$ \\
\hline CV $(\%)$ & 0,89 & 0,88 & 1,94 & 2,69
\end{tabular}

Médias seguidas da mesma letra não diferem significativamente pelo teste de Tukey. Fonte: Autores. 
Research, Society and Development, v. 11, n. 1, e20611124699, 2022

(CC BY 4.0) | ISSN 2525-3409 | DOI: http://dx.doi.org/10.33448/rsd-v11i1.24699

Tabela 3. Análise estatística NOx.

\begin{tabular}{ccccc}
\hline Tratamento & $\mathbf{1 , 0} \mathbf{k W}$ & $\mathbf{1 , 5} \mathbf{k W}$ & $\mathbf{4 , 5} \mathbf{k W}$ & $\mathbf{6 , 0} \mathbf{k W}$ \\
\hline B5 & $93,74 \mathrm{c}$ & $124,74 \mathrm{~b}$ & $213,71 \mathrm{c}$ & $256,58 \mathrm{e}$ \\
B10 & $107,35 \mathrm{a}$ & $132,97 \mathrm{a}$ & $213,42 \mathrm{c}$ & $260,23 \mathrm{de}$ \\
B15 & $81,32 \mathrm{~d}$ & $111,23 \mathrm{~d}$ & $230,13 \mathrm{a}$ & $264,74 \mathrm{c}$ \\
B20 & $101,29 \mathrm{~b}$ & $123,68 \mathrm{bc}$ & $214,06 \mathrm{c}$ & $261,77 \mathrm{ce}$ \\
B50 & $103,00 \mathrm{~b}$ & $123,61 \mathrm{bc}$ & $248,74 \mathrm{a}$ & $292,39 \mathrm{~b}$ \\
B100 & $96,45 \mathrm{c}$ & $121,68 \mathrm{c}$ & $249,35 \mathrm{a}$ & $301,35 \mathrm{a}$ \\
D100 & $81,16 \mathrm{~d}$ & $110,16 \mathrm{~d}$ & $204,29 \mathrm{c}$ & $236,71 \mathrm{f}$ \\
\hline CV (\%) & 5,63 & 3,22 & 6,81 & 2,39 \\
\hline
\end{tabular}

Médias seguidas da mesma letra não diferem significativamente pelo teste de Tukey. Fonte: Autores.

O desempenho geral do sistema motor gerador avaliado com biocombustíveis com base nos dados coletados de CE, NOx, CO e EE quando alimentado por biodiesel de canola, adicionado ao diesel na proporção de 10\% (B10) quando aplicada a carga de 4,5 kW, apresentou as menores emissões de CO (235,83 ppm), o menor consumo $\left(345,30\right.$ g.kW $\left.{ }^{-1} \mathrm{~h}^{-1}\right)$ e maior eficiência $(25,52 \%)$ do sistema de geração de energia para o motor gerador utilizado

\section{Conclusão}

O CE apresenta relação inversamente proporcional com as cargas resistivas, porém com decréscimos cada vez menores, conforme ocorre o aumento da carga. Houve aumento do $\mathrm{CE}$ à medida que aumentou a fração de biodiesel na mistura com o diesel, apresentando uma tendência decrescente no CE para as cargas de 1,0; 1,5 e 4,5 kW, com tendência crescente na carga de $6,0 \mathrm{~kW}$. Com o uso do biodiesel B100 no motor diesel, o CE aumentou quando comparado com o diesel tipo A. Com o acréscimo de cargas resistivas, o biodiesel apresenta um CE mais próximo do diesel, indicando que sua utilização em operações mais próximas da capacidade nominal é mais eficiente.

As emissões de $\mathrm{CO}$ e $\mathrm{CO}_{2}$ são inversamente proporcionais, enquanto as emissões de $\mathrm{CO}_{2}$ aumentam com a elevação de carga, o CO reduz, aumentando a carga. Os resultados indicaram que o uso do biodiesel em motores sem modificação é uma alternativa viável e ecologicamente correta. Porém, os testes indicaram um aumento nas emissões de NOx, em comparação ao diesel. Os valores também indicaram que as emissões de NOx são influenciadas pelo aumento de carga e pela composição das quantidades de biodiesel.

Levando-se em consideração os valores encontrados para os dados de emissões do motogerador e as cargas aplicadas durante os experimentos, observou-se que em sistemas alimentados por biocombustível não houve significativas reduções de $\mathrm{CO}_{2}$, porém como o $\mathrm{CO}_{2}$ emitido faz parte do ciclo da fotossíntese por ser de origem de culturas oleaginosas, as emissões globais não são aumentadas em comparação ao diesel.

A eficiência energética do motogerador obteve seu melhor resultado quando aplicada a carga de 4,5 kW, mostrando que cargas maiores podem ocasionar aumento de consumo de combustível.

Com base nos dados coletados (CE, NOx, $\mathrm{CO}, \mathrm{CO}_{2}$ e EE), vistas as metas de reduções dos gases de efeito estufa mundialmente acordados, o sistema utilizando biodiesel de canola, adicionado ao diesel de origem mineral na proporção de $10 \%$ (B10) quando aplicada a carga resistiva de 4,5 kW (75\% da carga nominal), apresentou as menores emissões, menor consumo e maior eficiência do sistema de geração de energia para o motor gerador utilizado.

Sugere-se para trabalhos futuros o desenvolvimento de pesquisas utilizando diferentes tipos de culturas oleaginosas que 
possibilitarão variados resultados, contribuindo na produção de energia sustentável, pensando na redução do uso de combustíveis de origem mineral.

\section{Referências}

Agarwal, A. K., Gupta, T., Dixit, N., \& Shukla, P. C. (2013). Assessment of toxic potential of primary and secondary particulates/ aerosols from biodiesel visà-vis mineral diesel fuelled engine. Inhalation Toxicology, 25(6), 325-332. 10.3109/08958378.2013.782515

Aldhaidhawi, M., Chiriac, R., Badescu, V., \& Delay, I. (2017). combustion and emission characteristics of Diesel engine fueled with rapeseed biodiesel - A literature review. Renewable and Sustainable Energy Reviews, 73, 178-186. 10.1016/j.rser.2017.01.129

Assefa, Y., Vara, P. V., Foster, C., Wright, Y., Young, S., Bradley, P. ... Ciampitti, I. A. (2018). Major management factors determining spring and winter canola yield in North America. Crop Science, 58(1), 1-16. 10.2135/cropsci2017.02.0079

Dharma, S., Haji, M., Chyuan, H., \& Hanra, A. (2017). Experimental study and prediction of the performance and exhaust emissions of mixed Jatropha curcasCeiba pentandra biodiesel blends in diesel engine using artificial neural networks. Journal of Cleaner Production, 164, 618-633. 10.1016/j.jclepro.2017.06.065

Erdo, S. (2020). Performance, emission and combustion characteristic assessment of biodiesels derived from beef bone marrow in a diesel generator. Energy, 207, 118300. 10.1016/j.energy.2020.118300

Gharehghani, A., Mirsalim, M., \& Hosseini, R. (2017). Effects of waste fi sh oil biodiesel on diesel engine combustion characteristics and emission. Renewable Energy, 101, 930-936. 10.1016/j.renene.2016.09.045

Hasan, M. A., Janius, R. B., Rashid, U., Taufiq-Yap, Y. H., Yunus, R., Zakaria, R., \& Mariah, N. (2015). Performance and exhaust emission characteristics of direct-injection diesel engine fueled with enriched biodiesel. Energy Conversion and Management, 106, 365-372, 2015. 10.1016/j.enconman.2015.09.050

Mohammad, S., Miri, R., Reza, S., Seyedi, M., \& Ghobadian, B. (2017). Effects of biodiesel fuel synthesized from non-edible rapeseed oil on performance and emission variables of diesel engines. Journal of Cleaner Production, 142, 3798-3808. 10.1016/j.jclepro.2016.10.082

Nayak, C., Pattanaik, B, P., \& Nayak, S. K. (2014). Effect of preheated jatropha oil and jatropha oil methyl ester with producer gas on diesel engine performance. International Journal of Automotive and Mechanical Engineering, 9, 1709-1722. 10.15282/ijame.9.2013.20.0142

No, S. (2011). How vegetable oils and their derivatives affect spray characteristics in ci engines - a review. Atomization and Sprays, 21(1), 87-105. 10.1615/AtomizSpr.v21.i1.60

No, S. (2014). Application of hydrotreated vegetable oil from triglyceride based biomass to CI engines - A review. Fuel, 115, 88-96, 2014. 10.1016/j.fuel.2013.07.001

Noor, C. W. M., Mamat, R., Najafi, G., Yasin, M. H. M., Ihsan, C. K., \& Noor, M. M. (2016). Prediction of marine diesel engine performance by using artificial neural network model. Journal of Mechanical Engineering and Sciences, 10(1), 1917-1930. 10.15282/jmes.10.1.2016.15.0183

Qi, D. H., Chen, H., Geng, L. M., \& Bian, Y. Z. (2010). Experimental studies on the combustion characteristics and performance of a direct injection engine fueled with biodiesel/diesel blends. Energy Conversion and Management, 51(12), 2985-2992. 10.1016/j.enconman.2010.06.042

Radha, K., Naga, S., Rajagopal, K., \& Nagesh, E. L. (2011). Performance and emission characteristics of a ci engine operated on vegetable oils as alternative fuels. International Journal of Automotive and Mechanical Engineering, 4, 414-427. 10.15282/ijame.4.2011.4.0034

Rahman, M. M., Rasul, M. G., Hassan, N. M. S., Azad, A. K., \& Uddin, M. N. (2017). Effect of small proportion of butanol additive on the performance, emission, and combustion of Australian native first- and second-generation biodiesel in a diesel engine. Environmental Science and Pollution Research, 24(28), 22402-22413. 10.1007/s11356-017-9920-6

Saifuddin, N., Refal, H., \& Kumaran, P. (2017). Performance and emission characteristics of micro gas turbine engine fuelled with bioethanol-diesel-biodiesel blends. International Journal of Automotive and Mechanical Engineering, 14(1), 4030-4049. 10.15282/ijame.14.1.2017.16.0326

Sakthivel, G., Nagarajan, G., Ilangkumaran, M., \& Bajirao, A. (2014). Comparative analysis of performance , emission and combustion parameters of diesel engine fuelled with ethyl ester of fish oil and its diesel blends. Fuel, 132, 116-124. 10.1016/j.fuel.2014.04.059

Serdar, H., Can, O., \& Ozt, E. (2017). Combustion and exhaust emissions of canola biodiesel blends in a single cylinder DI diesel engine. Renewable Energy, 109, 73-82. 10.1016/j.renene.2017.03.017

Severino, A. J. (2017). Metodologia do trabalho científico. São Paulo, SP: Cortez.

Simsek, S. (2020). Effects of biodiesel obtained from Canola, sefflower oils and waste oils on the engine performance and exhaust emissions. Fuel, $265,117026$. 10.1016/j.fuel.2020.117026

Singh, B., Kumar, N., Muk, H. (2012). A study on the performance and emission of a diesel engine fueled with Jatropha biodiesel oil and its blends. Energy, 37(1), 616-622. 10.1016/j.energy.2011.10.043

Sundararajan, K., Subbiah, G., \& Gomathinayakam, S. (2016). Emission estimation of neat paradise tree oil combustion assisted with superheated hydrogen in a 4-stroke natural aspirated dici engine. Thermal Science, 20, 1137-1145. 10.2298/TSCI16S4137S

Xue, J., Grift, T. E., \& Hansen, A. C. (2011). Effect of biodiesel on engine performances and emissions. Renewable and Sustainable Energy Reviews, 15(2), 1098-1116. 10.1016/j.rser.2010.11.016

Yesilyurt, M. K., \& Cesur, C. (2020). Biodiesel synthesis from Styrax officinalis L. seed oil as a novel and potential non-edible feedstock: A parametric optimization study through the Taguchi technique. Fuel, 265, 117025. 10.1016/j.fuel.2020.117025 